\title{
Nocturnal polyuria in men performing uroflowmetry for lower urinary tract symptoms
}

\author{
Emanuele Rubilotta ${ }^{1}$, Daniele Castellani ${ }^{2}$, Marilena Gubbiotti ${ }^{3}$, Matteo Balzarro ${ }^{1}$, \\ Giacomo Maria Pirola ${ }^{3}$, Rita Righetti ${ }^{4}$, Pierpaolo Curti ${ }^{4}$, Antonella Giannantoni ${ }^{5}$, \\ Maria Angela Cerruto ${ }^{1}$, Alessandro Antonelli ${ }^{1}$ \\ ${ }^{1}$ Urology Clinic, A.O.U.I of Verona, Verona, Italy; \\ 2 Department of Urology, IRCCS INRCA, Ancona, Italy; \\ ${ }^{3}$ Department of Urology, San Donato Hospital, Usl Toscana Sud-Est, Arezzo, Italy; \\ ${ }^{4}$ Department of Urology, AULSS 9, Ospedale Mater Salutis, Legnago, Italy; \\ ${ }^{5}$ Functional and Surgical Urology Unit, Department of Medical and Surgical Sciences and Neurosciences, University of Siena, \\ Siena, Italy.
}

\begin{abstract}
Summary Purpose: To assess the prevalence of nocturnal polyuria (NP) in males performing uroflowmetry (UF) for lower urinary tract symptoms (LUTS), the impact of NP on UF outcomes, and bladder emptying, the association between NP and LUTS.

Materials and methods: Men scheduled for UF were recruited in two Centres. Data collected were medical history, IPSS, UF, post-void residual urine volume (PVR), 3-day frequency-volume charts (FVC). The NP index was used to assess NP with a threshold of $\geq 33 \%$. The relationship between NP and patient's aging was assessed.

Results: 162 patients were included in the analysis. Mean age was $70.95 \pm 8.04$ years. The prevalence of NP was $54.9 \%$ (89/162). 110 (68\%) patients reported nocturia, and among these, NP was documented in 76 (69\%). Nocturia was found in $85 \%(76 / 89)$ of the population with NP. Total IPSS score, IPSS items \#1, \#2 and \#7 showed a significant difference in men with NP compared with those without. Maximum flow rate and PVR did not significantly change comparing men with or without NP. Mean voiding volume (VV) of the night-time micturitions was significantly higher in men with NP compared to those without $\mathrm{NP}(532.1 \pm 275.6 \mathrm{ml}$ vs $175 \pm 168.7 \mathrm{ml}$ respectively, $p<0001)$, while mean VV day-time micturitions and mean VV at UF did not change between groups.

Conclusions: NP had a high prevalence in men with LUTS performing UF. Aged males were more commonly affected by NP. Data demonstrated a strong relationship between NP and nocturia and increased urinary frequency while voiding symptoms were poorly related to NP.
\end{abstract}

KEY WORDS: Nocturnal polyuria; Nocturia; Uroflowmetry; Lower urinary tract symptoms; Male.

Submitted 12 January 2021; Accepted 5 March 2021

\section{INTRODUCTION}

The complaining of lower urinary tract symptoms (LUTS) is one of the most common causes that lead men to seek urological attention. Among LUTS, nocturia is one of the most bothersome (1) and it is frequently caused by nocturnal polyuria (NP), even in neurological patients (2-6). NP is a multifactorial disorder (7), defined by the International
Continence Society (ICS) as "passing large volume of urine during the main sleep period" $(8,9)$. Among men complaining of nocturia, the prevalence rate of NP is quite high, ranging from 76 to $88 \%$, and up to $93 \%$ in those aged $\geq 65$ years old ( 3 ). In a large community study, the prevalence of NP was confirmed high, with a prevalence of $77.8 \%$ of the whole studied population (9); NP has been demonstrated more prevalent in men with nocturia (91.9\%) compared to those without this condition (70.1\%) (10). Thereby, a lot of males complaining of nocturia might be affected by an underlying condition of NP, which might remain unidentified and consequently not adequately treated whether a bladder diary or a frequency volume chart (FVC) has not been used. Males with LUTS including nocturia, usually perform uroflowmetry (UF) as a first step assessment, since these symptoms are commonly considered suggestive of bladder outlet obstruction (BOO)/benign prostatic hyperplasia (BPH). In these subjects, NP is rarely investigated before the UF and, consequently, the prevalence of nocturia remains unknown. Similarly, also most commonly LUTS associated with men with an underlying NP condition who are candidates for UF is undetermined. NP mainly influences the bladder storage phase, and therefore this disorder is not expected to play an essential role in the voiding phase. However, there is still no conclusive data showing, in real-life practice, the rate of men with an underlying NP condition performing UF for LUTS and studies excluding with certainly an impact of NP on UF results. This study aimed to assess the prevalence of NP in males performing UF for LUTS and the influence of NP on UF outcomes. We also evaluated whether NP was significantly associated with nocturia, with other urinary symptoms investigated by IPSS questionnaire, and with the bother due to LUTS in males scheduled for UF. Finally, an analysis of the relationship between NP and patient's age was also performed.

\section{MATERIALS AND METHODS}

Between September 2017 and January 2019, all consecutive men with LUTS scheduled for UF execution were

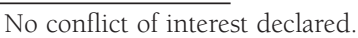


screened in outpatient clinics of two Tertiary Hospitals for study inclusion. The experimental procedures were carried out in accordance with the Declaration of Helsinki. This research was registered in our Department clinical audit. All patients signed written informed consent.

Data collected were a detailed medical history, selfadministered International Prostate Symptom Score (IPSS) questionnaire, UF, post-void residual urine volume (PVR) measured by ultrasound, and 3-day FVC, indicating "bedtime" and "waking time". The 3-day FVC was delivered to patients at the time the UF was scheduled and was returned by patients on the day of UF execution. Increased urinary frequency, urinary urgency, voided volume (VV), and nocturnal urine production were assessed by 3-day FVC. Nocturia was defined as at least one episode of nocturnal voiding at 3-day FVC. The nocturnal polyuria index (NPi) was used to assess NP (8). NPi is calculated by dividing the amount of nocturnal urine production by 24-hour production, and the threshold of $33 \%$ is the most accepted cut-off (8). Therefore, we used a cut-off of $\mathrm{NPi} \geq 33 \%$ of the total 24-h urine production to diagnose NP. Thus, patients were divided into two groups: Group A comprising males with $\mathrm{NPi}<33 \%$ (no NP patients) and was the control group, and Group B including men with NPi $\geq 33 \%$ (NP patients).

Inclusion criteria were age $\geq 18$ years, complete filling of both FVC and IPSS, UF with a voided volume higher than $150 \mathrm{~mL}$ as suggested by ICS recommendation on Good Urodynamic Practices (11). Exclusion criteria were all conditions that could be confounding factors, as following: surgery of lower urinary tract (prostatectomy, radical prostatectomy, radical cystectomy, bladder, and urethral surgery), pelvic radiation, urolithiasis, double J stent, and urinary devices, and recurrent lower urinary tract infection. The relationship between NP and maximum flow rate $\left(\mathrm{Q}_{\max }\right)$, VV at UF, and PVR after UF was evaluated. A comparison between the VV of the day-time and night-time micturitions was also assessed in each group. The VV of day-time and night-time micturitions and at UF were also compared between the two groups.

The association between NP and urinary symptoms was evaluated through the IPSS questionnaire, analyzing the IPSS total score and all IPSS item scores. According to the last "ICS report on the terminology for adult male lower urinary tract and pelvic floor symptoms and dysfunction", we divided the urinary symptoms reported by IPSS questionnaire as following: IPSS item \#1 -feeling of incomplete bladder emptying- as a "Postvoiding symptom"; IPSS item \#2 -increased urinary frequency, IPSS item \#4 urgency, IPSS item \#7 -nocturia- as "Storage symptoms"; IPSS item \#3 -intermittency, IPSS item \#5 -slow urinary stream, IPSS item \#6 -straining to void- as "Voiding symptoms" (9). The sub-analysis, according to patients' age, was also performed, distributing the patients into three groups according to age: 1) $<65$ years, 2) between 65 and 74 years, and 3$) \geq 75$ years.

\section{Statistical analysis}

Statistical analysis was performed with IBM-SPSS v. 17 for Windows (IBM Corp, Armonk, NY, USA). Student's t-test and the Mann- Whitney U test were performed to compare continuous parametric and nonparametric variables, as appropriate. Continuous variables were reported as mean \pm SD. All values in the text and tables are expressed as mean \pm SD. Statistically significant results were $p<$ or equal to 0.05 . Spearman correlations were used to test for the strength of linear association between variables along with the Wilcoxon and Mann-Whitney. $X^{2}$ test was also used. Multiple linear regression analysis was performed evaluating NP and the following variables: NPi, total nocturnal diuresis, total daily (24 hours) diuresis, $\mathrm{Q}_{\max ,}$ PVR, age, cardiologic disease and diuretic drugs.

\section{RESULTS}

During the study period, 254 men met inclusion criteria and were enrolled in the study. Data on UF, PVR, and both IPSS and 3-day FVC were completed in 162/254 (63.8\%) patients, who were included in the analysis. Table 1 shows the patients' characteristics. Mean age was $70.95 \pm$ 8.04 years. Among males aged lower than 65 years (Group i), one patient was 47 years old, and the remaining were older than 52 years. Eighty-eight (54.3\%) patients were on urological therapy and $44(27.1 \%)$ on cardiological treatment. Only 15 (9.2\%) patients were on diuretics, and 7 (4.3\%) were diabetic.

The prevalence of NP was 54.9\% (89/162 patients) with a mean NPi of $34.4 \pm 11.2$. Table 2 reports the rate of NP according to age, and Table 3 the comparison between the groups stratified by age. Younger males were the smaller group ( $\mathrm{n}=34$ ), but with the highest NP rate $(64.7 \%)$. $75 \%$ of the men affected by NP were aged $\geq 65$ years old. Finally, we found a positive association between NP and age only in the group of men 65-74 years-old ( $<0.05$ ). One-hundred-ten (68\%) patients reported nocturia, and among these, NP was documented in 76 (69\%). Nocturia was found in $85 \%(76 / 89)$ of the population with NP. Total IPSS score, IPSS items \#1, IPSS items \#2 and \#7 showed a significant difference in men with NP compared with those without this disorder (Table 4). The maximum flow rate and PVR did not significantly change in the two groups of males (Table 4).

Multiple regression analysis showed a significant correlation between NP and NPi, total nocturnal diuresis, total $24 \mathrm{~h}$ daily diuresis, age, cardiologic disease, and diuretic drugs $(r=0.96 ; p=0.001)$, but not with $Q_{\max }$ and PVR $(\mathrm{r}=2 ; \mathrm{p}=0.8)$.

Table 1.

Patients' characteristics.

\begin{tabular}{|c|c|}
\hline Patients ( $n=162$ ) & $n(\%)$ \\
\hline $\begin{array}{l}\text { Patients in cardiological therapy } \\
\text { - diuretic therapy } \\
\text { - non diuretic therapy for hypertension } \\
\text { - antiarrhythmics } \\
\text { - antiplatelets/anticoagulants } \\
\text { - diabetes therapy }\end{array}$ & $\begin{array}{c}4(27.1) \\
15(9.2) \\
24(14.8) \\
9(5.5) \\
11(6.7) \\
7(4.3)\end{array}$ \\
\hline $\begin{array}{l}\text { Patients in urological therapies } \\
-\alpha 1 \text {-blockers } \\
-5 \alpha \text {-reductase inhibitors } \\
-\alpha 1 \text {-blockers }+5 \alpha \text {-reductase inhibitors } \\
\text { - phytotherapy }\end{array}$ & $\begin{array}{c}88(54.3) \\
62(38.3) \\
25(15.4) \\
11(6.8) \\
1(0.6)\end{array}$ \\
\hline
\end{tabular}


Table 2.

Nocturnal polyuria prevalence according to patients' age.

\begin{tabular}{|l|c|c|}
\hline Age (years) & $\begin{array}{c}\mathbf{N P i}<\mathbf{3 3} \% \\
(\mathbf{n}=\mathbf{7 3})\end{array}$ & $\begin{array}{c}\mathbf{N P i} \geq \mathbf{3 3} \% \\
(\mathbf{n}=\mathbf{8 9})\end{array}$ \\
\hline$<65(\mathrm{n}=34)$ & $12(35.3)$ & $22(64.7 \%)$ \\
\hline $65-74(\mathrm{n}=66)$ & $34(51.6 \%)$ & $32(48.4 \%)$ \\
\hline$\geq 75(\mathrm{n}=62)$ & $27(43.6 \%)$ & $35(56,4 \%)$ \\
\hline Tot $\mathrm{n}=162$ & $73(45.0 \%)$ & $89(55 \%)$ \\
\hline NPi: nocturnal polyuria index. & \\
\hline
\end{tabular}

Table 3.

Comparison between patients with NPi > 33\% stratified by age.

\begin{tabular}{|l|c|c|c|c|}
\hline $\mathrm{NPi} \geq \mathbf{3 3} \%$ & $<\mathbf{6 5}(\mathrm{n}=\mathbf{3 4})$ & $\mathbf{6 5 - 7 4}(\mathrm{n}=\mathbf{6 6})$ & $\geq \mathbf{7 5}(\mathrm{n}=\mathbf{6 2})$ & $\mathbf{P}$ \\
\hline & $22(64.7 \%)$ & $32(48.4 \%)$ & $\cdot$ & 0.4 \\
\hline & - & $32(48.4 \%)$ & $35(56.4 \%)$ & 0.05 \\
\hline & $22(64.7 \%)$ & - & $35(56.4 \%)$ & 0.5 \\
\hline
\end{tabular}

\section{Table 4.}

Correlations between NP and IPSS scores, maximum flow rate, and post-void residual urine volume.

\begin{tabular}{|c|c|c|c|c|}
\hline & & $\mathrm{NPi}<33 \%(\mathrm{n}=73)$ & $\mathrm{NPi} \geq 33 \%(\mathrm{n}=89)$ & $\mathbf{P}$ \\
\hline \multirow[t]{9}{*}{ IPSS score, mean \pm SD } & Item \# 1 & $1.0 \pm 1.5$ & $1.2 \pm 1.6$ & 0.002 \\
\hline & Item \# 2 & $1.0 \pm 1.1$ & $1.6 \pm 0.9$ & 0.00 \\
\hline & Item \# 3 & $0.9 \pm 1.4$ & $1.4 \pm 1.8$ & 0.4 \\
\hline & Item \# 4 & $0.7 \pm 1.2$ & $0.7 \pm 1.4$ & 1 \\
\hline & Item \# 5 & $1.3 \pm 1.5$ & $1.6 \pm 1.9$ & 0.07 \\
\hline & Item \# 6 & $0.4 \pm 0.8$ & $0.6 \pm 1.3$ & 0.2 \\
\hline & Item \# 7 & $1.5 \pm 0.4$ & $2.5 \pm 0.7$ & 0.00 \\
\hline & Item \# 8 & $1.9 \pm 0.8$ & $1.9 \pm 1.1$ & 0.06 \\
\hline & Total & $6.5 \pm 3.5$ & $9.8 \pm 6.7$ & 0.00 \\
\hline \multicolumn{2}{|l|}{$\overline{Q_{\text {max }}(\text { mean } \pm S D)}$} & $13.4 \pm 2.5$ & $11.6 \pm 2.6$ & 0.08 \\
\hline \multicolumn{2}{|l|}{$\overline{P V R}($ mean $\pm S D)$} & $40.05 \pm 30.2$ & $37.8 \pm 20.9$ & 0.2 \\
\hline
\end{tabular}

\section{Table 5.}

Comparison between voiding volume of day-time and nighttime micturition and voiding volume on uroflowmetry stratified according to Nocturnal polyuria index.

\begin{tabular}{|l|c|c|c|}
\hline & $\mathbf{N P i}<\mathbf{3 3} \% \mathbf{( n = 7 3 )}$ & $\mathbf{N P i} \geq \mathbf{3 3} \% \mathbf{( n = 8 9 )}$ & $\mathbf{P}$ \\
\hline W of night-time micturition, mean \pm SD & $175.2 \pm 168.7$ & $532.1 \pm 275.6$ & 0.0001 \\
\hline W of day-time micturition, mean \pm SD & $222.1 \pm 83.1$ & $213.7 \pm 79.4$ & 0.5 \\
\hline W at UF, mean \pm SD & $267.1 \pm 136.3$ & $246.7 \pm 109.7$ & 0.2 \\
\hline NPi: nocturnal polyuria index; WV: voding volume.
\end{tabular}

Table 5 reports data on the comparison of the mean VV of the micturitions at night-time, day-time, and at UF between the two groups. Mean VV of the night-time micturitions was significantly higher in men with the diagnosis of NP compared to those without NP recorded at FVC $(\mathrm{p}<0001)$. Mean VV of the micturitions during the daytime and measured at UF was not statistically different between the two groups. However, volumes were slightly higher in Group A. In Group A, the mean VV of micturitions at day-time was $222.1 \pm 83.1 \mathrm{ml}$, while at night- time was $175.2 \pm 168.7(p=0.03)$. Conversely, in Group B mean VV of micturitions at day-time was significantly lower than at night-time, $213.7 \pm 79.4 \mathrm{ml}$, and $532.1 \pm$ $275.6 \mathrm{ml}$ respectively $(\mathrm{p}=0.0001)$.

Total daily (24-hours) diuresis was $757.8 \pm 73.7 \mathrm{ml}$ in Group A, and 1095.6 $\pm 103.8 \mathrm{ml}$ in Group B (p < 0.0001). Total nocturnal diuresis was $223.8 \pm 64.1 \mathrm{ml}$ in Group A, and $587.1 \pm 86.6 \mathrm{ml}$ in Group B $(\mathrm{p}<0.0001)$. The mean nocturnal number of voids was $0.8 \pm 0.7$ in Group $\mathrm{A}$, and $1.9 \pm 1$ in Group B $(\mathrm{p}<0.0001)$.

\section{Discussion}

To date, the prevalence of NP in men candidates to UF for LUTS, and the impact of NP on bladder emptying, have been poorly investigated (12).

Our study demonstrated a high NP prevalence (54.9\%) in men performing UF for LUTS. In this cohort, NP was a common condition, identified by FVC in more than half of the cases. The rate of NP was lower than the data reported in community studies $(10,13)$. Nevertheless, it was comparable to the NP prevalence reported in the Caucasian population, and cohorts of men with LUTS (1416). The high rate of NP in this selected population of men performing UF for LUTS may be explained by the age and comorbidities of the patients, and by the coexistence of NP with other pathological conditions, such as BOO/BPH or overactive bladder (OAB) syndrome, which may cause LUTS. Another reason could be that NP, due to the related LUTS, in some cases could be confused with other clinical conditions needing an investigation with UF.

In our series, NP had a relevant association with most of the storage symptoms, mainly nocturia, and with postvoiding symptoms, but not with voiding symptoms. At the same time, the parameters related to voiding and bladder emptying, such as $\mathrm{Q}_{\max }$ and $\mathrm{PVR}$, did not change significantly between the two groups of patients. Therefore, in our cohort, data on LUTS and UF demonstrated that NP had a significant impact on the bladder filling phase, but only a limited effect on the voiding phase. Men who referred mainly storage symptoms, complaining mostly of nocturia, and reported only a few voiding/postvoiding symptoms, were the most likely patients to be affected by NP. These males should be investigated with FVC in addition to symptomatic questionnaires, and to UF with PVR, to avoid misleading diagnosis, useless examinations, and treatments. Symptomatic questionnaires, as IPSS, are useful to define the patient's LUTS, but they are not able to identify NP. These tools cannot assess whether nocturia was a symptom correlated to $\mathrm{BOO} / \mathrm{BPH}$ or $\mathrm{OAB}$, or, conversely, to the large amount of nocturnal urine which, continuously filling the bladder, leads the patient to awake. This latter pathophysiological mechanism was demonstrated by our data, showing that the mean VV of night-time micturitions was significantly higher in men with nocturia and NP. In males with unidentified NP, when nocturia is mistakenly evaluated as a $\mathrm{BOO} / \mathrm{BPH}$ or OAB symptom, inappropriate therapies with alpha-blockers or anticholinergics agents may be offered to patients. The outcomes of these latter treatments have been reported as unsuccessful on NP and poorly effective on nocturia, and thus should be avoided $(3,12,16)$.

In men with $\mathrm{BOO} / \mathrm{BPH}$, usually voiding symptoms are 
prevalent, and abnormal UF parameters are often associated with this condition (17). Conversely, our data showed that men with NP had no voiding symptoms, and both $\mathrm{Q}_{\max }$ and PVR were not associated with NP, because the UF outcomes did not significantly differ between men affected by NP and males without NP. These results support the finding that NP is scarcely related to voiding dysfunctions, as was also supposed by a study on a small sample size of men with LUTS suggestive of BPH performing UF (12). In this small population, the rate of NP was $95 \%$, and in $75 \%$ of these patients, NP did not significantly vary after alpha-blockers therapy, although an increase in $Q_{\max }$ was found in the treated men (12) Hence, males with NP may have concomitant $\mathrm{BOO} / \mathrm{BPH}$, but usually, the latter is not the leading cause of NP. For this reason, conventional $\mathrm{BOO} / \mathrm{BPH}$ treatments often poorly influence NP conditions.

The other pathological condition which should be distinguished from NP is represented by OAB syndrome, defined by "urinary urgency, usually accompanied by increased daytime frequency and nocturia, with or without urinary incontinence" (9). In our cohort, NP was significantly associated with increased urinary frequency and nocturia, but not with urinary urgency, which is one of the most characterizing symptoms of OAB (9).

Furthermore, $\mathrm{OAB}$ syndrome is often accompanying by a reduced VV, which was not observed in NP patients of our study. Finally, the reporting of mainly storage symptoms, as frequency and nocturia, without urgency, and with no reduced VV at FVC, might be considered as a "red-flag" for differentiating between $\mathrm{OAB}$ and NP.

An accurate assessment of LUTS and FVC, in addition to the other first-step office evaluations, may aid in the achievement of a correct differential diagnosis.

Our study confirmed previous data on the very close relationship between nocturia and NP $(2-4,14,15)$.

The majority of the males with NP reported concomitant nocturia ( $85 \%$ ), due to the increased nocturnal urine production, which led them to awake. On the other hand, patients reporting nocturia also demonstrated a high rate of NP (69\%). Therefore, in males affected by nocturia, a concomitant NP should be supposed and should be investigated appropriately with FVC. Furthermore, our study confirmed that, also in men performing UF for LUTS, severe nocturia associated with VV significantly lower at day-time than at night-time might warn on a condition of NP (18).

Feeling of incomplete bladder emptying, a postvoiding symptom, was significantly associated with NP. Patients affected by NP showed a higher severity of frequency and nocturia. Therefore, men with NP might be more concerned by the increased need to urinate, and consequently, they may have a greater sensation of not having completely emptied the bladder. Furthermore, in men with NP, the mean VV of the night-time micturitions was more than twice compared to the mean VV of the day-time, and significantly higher than the mean VV of micturitions at night of patients with no NP. The excessive night-time urine production may cause recurrent nocturnal overstretching of detrusor fibers, as the high nocturnal VV documented, leading patients to a worse bladder emptying and, as a consequence, to a greater feeling of incomplete bladder emp- tying. Moreover, the increased night-time bladder capacity may amplify the feeling of bladder filling even after micturition. These reasons may also explain the higher rate of increased urinary frequency in NP males.

The quality of life (IPSS item \#8) was not significantly associated with NP in our study. Patients enrolled during a UF, might have been more concerned by micturition and bladder emptying, which are poorly influenced by NP. This could be a possible explanation of this finding and could be a limit of our study.

NP was more prevalent in younger males, but this result may be related to the low number of younger men included in our study. Indeed, we found that NP increased with age, showing that $75 \%$ of men with NP were aged $\geq 65$ years old. Data also showed a significant association between NP rate and age in the older males, although not in the oldest ( $>75$ years). The relationship between NP and aging might be influenced by population selection, race, NPi criteria, and sample size. Most of the literature data on NP have been reported considering the general population or in community cohorts. Conversely, our cohort included only males with LUTS performing UF, with strict inclusion/exclusion criteria, and these parameters may have influenced our findings on aging.

The strength of our study was the finding of the relevant prevalence of NP on a large sample size of males performing UF for LUTS. Our data also demonstrated that NP has a limited impact on micturition and bladder emptying. These latter data could only have been hypothesized, but not documented, before our study. Another strong point of our research was the identification, among the main storage symptoms, of those significantly associated with men with NP who perform UF for LUTS, and the uncovering of the voiding/postvoiding symptoms most commonly reported by these patients. Hence, the value of our study was to recognize the "warning symptoms" of men with NP, among the males scheduled for the UF for LUTS.

A limit of our study was the lack of repeated UFs, due to the real-practice design of the present research. Another limit was the low sample size of the younger people, which was smaller than the other age groups. The choice of the NPi threshold of $33 \%$ might be a further limitation of the study. However, we have used this cut-off because it is one of the most accepted. Furthermore, there were only a few younger males in our study, and a lower threshold could have been useful in cohorts comprising younger patients than those enrolled in our study. A further limitation of the research is that NP has been correlated only with the urinary symptoms reported by the IPSS questionnaire, and not with all the LUTS. However, these symptoms are the most commonly complained by the males and assessed by clinicians. The strict inclusion/exclusion criteria led to reducing the number of males recruited in the study.

The choice of these stringent parameters may have been a limit of this research, but allowed us to reduce potential biases and achieve more reliable data on the prevalence and impact of NP in men with LUTS performing UF.

\section{Conclusions}

In conclusion, our study highlighted that urologists should always look for NP among men who report noc- 
turia and mainly storage symptoms, and only mild emptying/postvoiding symptoms. Both IPSS and FVC are needed to diagnose NP accurately, while UF with PVR assessment is useful for excluding potential associated pathological conditions.

\section{REFEREnCES}

1. Agarwal A, Eryuzlu LN, Cartwright R, et al. What is the most bothersome lower urinary tract symptom? Individual- and population-level perspectives for both men and women. Eur Urol 2014; 65:1211-17.

2. Fujimura T, Yamada Y, Sugihara T, et al. Nocturia in men is a chaotic condition dominated by nocturnal polyuria. Int J Urol 2015; 22:496-501.

3. Weiss JP, Van Kerrebroeck PEV, Klein BM, Nørgaard JP. Excessive nocturnal urine production is a major contributing factor to the etiology of nocturia. J Urol 2011; 186:1358-63.

4. Chang SC, Lin ATL, Chen KK, Chang LS. Multifactorial nature of male nocturia. Urology 2006; 67:541-4.

5. Haddad R, Denys P, Arlandis S, et al. Nocturia and nocturnal polyuria in neurological patients: from epidemiology to treatment. A systematic review of the literature. Eur Urol Focus 2020; 6:922-34.

6. Birder LA, Van Kerrebroeck PEV. Pathophysiological mechanisms of nocturia and nocturnal polyuria: the contribution of cellular function, the urinary bladder urothelium, and circadian rhythm. Urology 2019; 133:14-23.

7. Cornu JN, Abrams P, Chapple CR, et al. A contemporary assessment of nocturia: Definition, epidemiology, pathophysiology, and management - A systematic review and meta-analysis. Eur Urol. 2012; 62:877-90.

8. Van Kerrebroeck P, Abrams P, Chaikin D, et al. The standardisation of terminology in nocturia: Report from the standardisation sub- committee of the international continence society. Neurourol Urodyn. 2002; $21: 179-83$

9. D'Ancona C, Haylen B, Oelke $M$, et al. The International Continence Society (ICS) report on the terminology for adult male lower urinary tract and pelvic floor symptoms and dysfunction. Neurourol Urodyn. 2019; 38:433-77.

10. Van Doorn B, Blanker MH, Kok ET, et al. Prevalence, incidence, and resolution of nocturnal polyuria in a longitudinal community-based study in older men: The Krimpen study. Eur Urol. 2013; 63:542-7.

11. Gammie A, Drake MJ. The fundamentals of uroflowmetry practice, based on International Continence Society good urodynamic practices recommendations. Neurourol Urodyn. 2018; 37: S44-S49.

12. Koseoglu H, Aslan G, Ozdemir I, Esen A. Nocturnal polyuria in patients with lower urinary tract symptoms and response to alphablocker therapy. Urology 2006; 67:1188-92.

13. Swithinbank L V, Vestey S, Abrams P. Nocturnal polyuria in community-dwelling women. BJU Int 2004; 93:523-7.

14. Mariappan P, Turner KJ, Sothilingam S, et al. Nocturia, nocturia indices and variables from frequency-volume charts are significantly different in Asian and Caucasian men with lower urinary tract symptoms: A prospective comparison study. BJU Int. 2007; 100:332-6.

15. Klingler HC, Heidler H, Madersbacher H, Primus G. Nocturia: An Austrian study on the multifactorial etiology of this symptom. Neurourol Urodyn. 2009; 28:427-31.

16. Yoong HF, Sundaram MB, Aida Z. Prevalence of nocturnal polyuria in patients with benign prostatic hyperplasia. Med J Malaysia. 2005; 60:294-6.

17. Thorner DA, Weiss JP. Benign prostatic hyperplasia: symptoms, symptom scores, and outcome measures. Urol Clin North Am. 2009; 36:417-29.

18. Presicce F, Puccini F, De Nunzio C, et al. Variations of night-time and daytime bladder capacity in patients with nocturia: implication for diagnosis and treatment. J Urol. 2019; 201:962-6.

\author{
Correspondence \\ Emanuele Rubilotta, MD \\ emanuele.rubilotta@aovr.veneto.it \\ Matteo Balzarro, MD \\ matteo.balzarro@aovr.veneto.it \\ Maria Angela Cerruto, MD (Corresponding Author) \\ mariaangela.cerruto@univr.it \\ Alessandro Antonelli, MD \\ alessandro.antonelli@univr.it \\ Urology Clinic, A.O.U.I of Verona, Verona (Italy) \\ Daniele Castellani, MD \\ d.castellani@inrca.it \\ Department of Urology, IRCCS INRCA, Ancona (Italy) \\ Marilena Gubbiotti, MD \\ marilena.gubbiotti@gmail.com \\ Giacomo Maria Pirola, MD \\ giacomomaria.pirola@uslsudest.toscana.it \\ Department of Urology, San Donato Hospital, Usl Toscana Sud Est, Arezzo (Italy) \\ Rita Righetti, MD \\ rita.righetti@aulsslegnago.it \\ Pierpaolo Curti, MD \\ pierpaolo.curti@aulss9.veneto.it \\ Department of Urology, AULSS 9, Ospedale Mater Salutis, Legnago (Italy) \\ Antonella Giannantoni, MD \\ antonella.giannantoni@unisi.it \\ Functional and Surgical Urology Unit, Department of Medical and Surgical Sciences \\ and Neurosciences, University of Siena, Siena (Italy)
}

